Copyright@ I.C. Baianu, 2004.

Bulletin of Mathematical Biophysics, $\underline{33}: 349-365$ (1971).

\title{
ORGANISMIC SUPERCATEGORIES AND QUALITATIVE DYNAMICS OF SYSTEMS
}

\author{
I. Baianu* \\ University of Bucharest, \\ Faculty of Physics, \\ Dept. of Electricity and Biophysics, \\ Str. Academiei nr. 14, Bucharest, \\ Romania
}

*Currently at The University of Illinois at Urbana, Urbana, Illinois 61801, USA.

\section{Abstract.}

The representation of biological systems by means of organismic supercategories, developed in previous papers, is further discussed. The different approaches to relational biology, developed by Rashevsky, Rosen and by Baianu and Marinescu, are compared with Qualitative Dynamics of Systems which was initiated by Henri Poincaré (1881). On the basis of this comparison some concrete results concerning dynamics of genetic system, development, fertilization, regeneration, dynamic system analogies, and oncogenesis are derived.

1. Introduction. In previous papers (Baianu and Marinescu, 1968; Comorozan and Baianu, 1969; Baianu, 1970; herein afterwards referred to as I, II, III, respectively), a categorical representation of biological systems was introduced. This representation is different from Rosen's categorical approach to relational biology (Rosen 1958a, b; 1959).

The aim of this paper is to present some concrete results which are derived on the basis of our representation. We shall reach finally an idea which was advanced by Rashevsky fourteen years ago, namely, that the geometrization of physics suggests a possible geometrization of biology. However, we suggest that the necessary improvement to be made is that of developing specific 
techniques from Algebraic Geometry. The basic concept of our theory is that of an organismic supercategory which is a categorical formalization of Rashevsky's notion of an organismic set. The mathematical ideas underlying this concept are those of structure and generator. They were largely discussed in III. Nevertheless, in order to maintain a self contained presentation, I shall emphasize here some of the basic aspects of our representation. Let us consider a system whose state space consists of a torus such that the states of the system are contained inside the torus, and all transitions lead to states inside the torus. The homology theory offers in this case two intuitive examples of generators. The whole torus is generated by two cycles only, and these cycles are shown in Figure 1, as dotted circles.

\section{FIGURE 1}

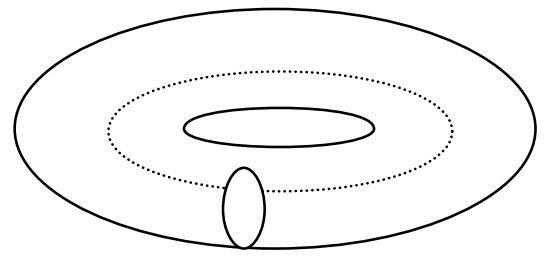

The two cycles generate two homology groups $H_{\mathbf{o}}(T)=Z$ and $H_{1}(T)=Z \mathbb{Z}$, where $Z$ is the group of integers, and $\oplus$ denotes the product operation. These homology groups give a characterization of the topological space represented by the torus. In this.way a connection is established between a topological structure and algebraic structures. Even more, we can assign two numbers to a given complex K: the Betti number-which is the number $\mathrm{L}$ of repetitions of $\mathrm{Z}$ in the homology group, $\mathrm{H}_{\mathbf{p}}(\mathrm{K})=\mathrm{Z} \oplus \mathrm{Z} \oplus \ldots \oplus \mathrm{Z} \oplus \mathrm{GPT}$ of a complex $\mathrm{K}$-and a number $\mathbf{p}$ which is the number of elements of a finite abelian group GPT. The Betti number gives the number of $\boldsymbol{p}$ dimensional holes of the complex $\mathrm{K}$, and the number $\mathbf{p}$ gives the number of p-dimensional turns of $\mathrm{K}$.

The physical interpretation which we shall give to the holes inside of the state space of a system will be that of instability fields of the system under consideration. Consequently, the 
Betti number will give a coarse idea about the instability of the system, being the number of instability fields of the state space. However, homological techniques would allow a fine characterization of the local and global properties of dynamics of a system, being able to locate singularities in a state space (Hwa and Teplitz, 1966). It must be mentioned here that the theory of categories and functors gives a further improvement of homological techniques. In the above discussed example a number was assigned to a quality, that is, $\boldsymbol{a}$ Betti number was assigned to a topological space. Another example of such an assignment is found in the theory of elementary particles, where one associates a probability with a Feynman diagram. A simple and intuitive example is shown in Figure 2:

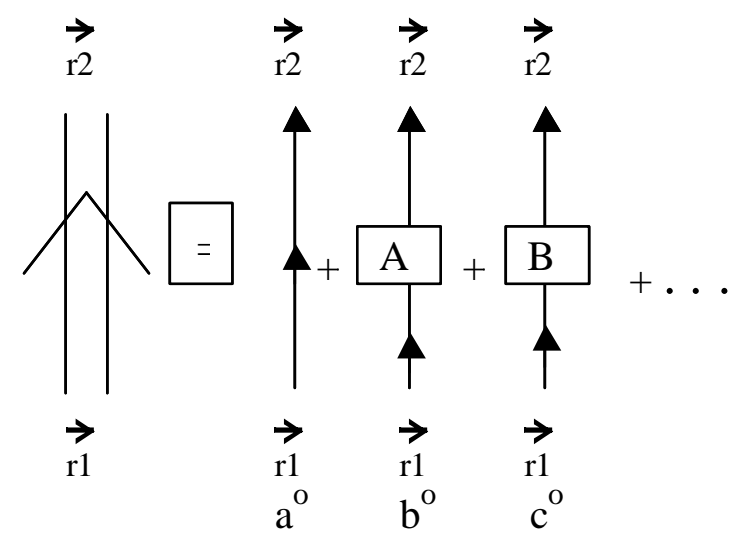

Figure 2. (Explanations are given in text)

A particle which is moving from a point $\vec{r}$ of the space towards a point $\vec{r} 2$ is subject to a number of interactions in regions $\mathrm{A}, \mathrm{B}, \ldots$ of space. The probability that a given particle would have a free way (that is, without interactions from $\overrightarrow{\mathrm{r}} \overrightarrow{\mathrm{t}}$ to $\mathrm{r} \overrightarrow{\text { ) }}$ corresponds to diagram a) from above.

The probability that a given system would interact in region A corresponds to diagram b), etc. Thus, from the above diagrams we may compute the Green function $\mathrm{G}(\overrightarrow{\mathrm{r} 2}, \mathrm{t} 2 ; \overrightarrow{\mathrm{r}}, \mathrm{t} 1)$ of a particle (the Green function of a particle is defined as the probability that the particle would reach the point $\mathrm{r} 2$, at moment $\mathrm{t} 2$, coming from the point $\mathrm{r} 1$, where it was at $\mathrm{t} 1$ ). 
However, the general procedure is not to assign a single number to a quality, but an entire set of numbers. In our second example in Figure 2, the operation of addition induced a corresponding operation on diagrams. This fact suggests that operations which are used in metric, or quantitative, biology may induce corresponding operations in relational biology. Conversely, one can think of significant relational operations with notions, or concepts, which would permit us to obtain solutions to complicated problems of quantitative biology. Throughout this paper we shall make extensive use of this basic idea.

\section{Observables, Generators and Qualitative Dynamics.}

In III we suggested a general definition of observables as morphisms, or as functors. Observables of a biological system may be introduced as intensities of some activities of the whole system, as parameters characterizing processes inside the system, or as variables which specify the quantities of certain products which are formed as a result of activities of the system. Among observables, structural parameters (Rosen 1968a, b) and time observables play a distinguished role. Some observables are "linked," that is, a change in one of them implies a corresponding change in all the others. Linked observables were represented as morphisms in a diagram. This diagram corresponds to the linkage group of observables and is a part of the

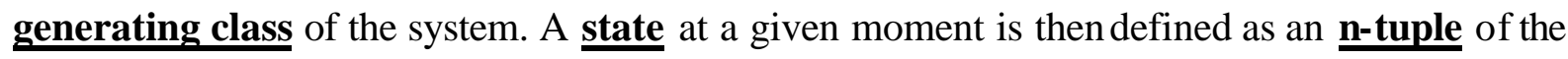
values of essential observables at that moment. In our representation, a state is defined as a functor from the category of generating classes of the system to $\mathbf{R}$--the set of real numbers organized as a discrete category (or as a category whose objects are real numbers, and whose morphisms are mappings; the operations with real numbers in this category are induced by the structure of the category of generating classes). Let us consider a specific example. An operon (Jacob and Monod, 1961) may be considered as having two states: an active state and an inactive one. In its inactive state the operon will not induce the synthesis of the corresponding enzyme, while in its active state, it will induce the synthesis of a determined quantity $\mathrm{C}$ of synthesized enzyme per unit of time. Now, if we consider a linkage group of operons $\mathrm{O}_{1}, \mathrm{O}_{2}, \ldots, \mathrm{O}_{\mathrm{n}}$, which are all active in the same time and, if the synthesized quantities of enzymes per unit of time are respectively, $\mathrm{C}_{1}, \mathrm{C}_{2}, \ldots, \mathrm{C}_{\mathrm{n}}$, a state of the linked operons may be defined by the $\mathrm{n}$-tuple $\left(\mathrm{C}_{1}^{\mathrm{t}}\right.$, 
$\mathrm{C}_{2}^{\mathrm{t}}, \ldots, \mathrm{C}_{\mathrm{n}}^{\mathrm{t}}$ ) of the values of $\mathrm{C}_{1}, \mathrm{C}_{2}, \ldots, \mathrm{C}_{\mathrm{n}}$ at the given moment $\mathrm{t}$. However, suppose that only $\mathrm{C} 1$ ' $\mathrm{C} 2$ and $\mathrm{Ca}$ are essential, all other observables being expressed in terms of $\mathrm{C}_{1}, \mathrm{C}_{2}$ and $\mathrm{C}_{3}$. Even more, let us suppose that we may find some operators such that $\mathrm{C}_{2}=\mathrm{YC}_{1}$ and $\mathrm{C}_{3}=\mathrm{ZC}_{2}$. In this case there exists a third operator $\mathrm{X}$ such that $\mathrm{C}_{3}=\mathrm{XC}_{1}$, and such that the following diagram will be commutative.

\section{Diagram 1:}

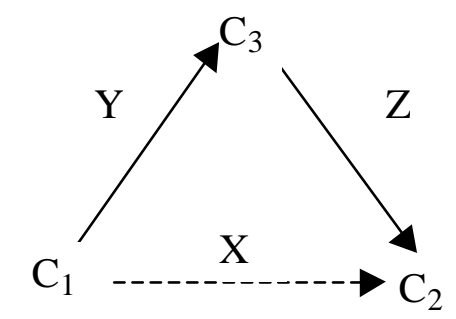

The above diagram is the generating diagram of the linkage group of operators. According to our representation, a genetic system will be then represented by a generating class, whose objects are generating diagrams of the linkage groups of operons, and whose morphisms are the functional connections among the activities of the operons. Suppose that a mutation takes place in the genetic system, such that an operon will begin to induce the synthesis of an enzyme which was not synthesized previously by the system. The state corresponding to the very moment when the change takes place will be considered as a singularity of the state space, as far as in that moment one cannot characterize the state of the genetic system either by $\mathrm{C}_{\mathrm{k}}^{\mathrm{t}}$-the quantity of synthesized enzyme $E_{k}$ per unit of time, or by $\mathrm{C}^{, \mathrm{t}}{ }_{\mathrm{k}}$ the quantity of the new enzyme $E{ }_{k}$ per unit of time (that is, $E^{\prime}{ }_{k}$ is the enzyme which begins to be synthesized after the mutation took place). It may happen that a mutation produces effects such as the complete inactivation of an essential operon. In this case, it is conceivable that the whole linkage group will become inactive. If the 
inactivated operon is the replicon, or "replicone" (Jacob et al., 1963), then the cell will cease to divide. On the other hand, if such a mutation, or sequence of mutations, involved the inactivation of a linkage group of tumor suppressor genes, the cell may transform into a malignant cell, and thus may cause cancer. The corresponding singularity of the state space in the case of mutation would last much less than other states of the dynamical system, and according to definition (D8) of III (p. 556), may be considered as an unstable state inside the state space of the system. Generally, if the unstable state leads only to other unstable states, it may result in the destruction of the system generating an unstable field, thus lying outside the state space of the system. Consequently, states in growth processes should have to be considered as metastable and must not be considered as short-lived, or unstable.

The replacement of an observable by another, in case of mutation, is in fact a change of the structure of the genetic system. Insofar as the dynamical system is defined as an input-output device with a determined structure (Rosen 1958a, 1968b), or as a couple $\left(\mathrm{S},\left\{f_{\mathrm{t}}\right\}\right)$, (Rosen, 1968a), we should have to consider a mutation as a transformation of the system into another system. In our representation, it will be justified only when the generating class is affected by the mutation, that is, only when an essential observable is replaced by another observable, which did not belong to the system.

Thus, as in III, we consider a dynamical system D to be a commutative diagram with: X-the "state space" of D (that is, a supercategory the objects of which are states, and morphisms of which are transitions among states or fields of states), $\mathrm{R}^{\mathbf{n}}$-the category whose objects are elements of $\mathrm{R}, \mathrm{R} \times \mathrm{R}=\mathrm{R}^{2}, \ldots, \mathrm{R} \times \mathrm{R} \times \ldots \times \mathrm{R}=\mathrm{R}^{\mathrm{n}}$, and whose morphisms are operators on real numbers or functions, $\bar{S}$-the supercategory of generating classes and morphisms among these, and $\bar{T}$-the "time supercategory," that is a supercategory such that the structure of $\bar{S}$ mainly depends on the structure of $\bar{T}$ by setting a one-to-one correspondence 


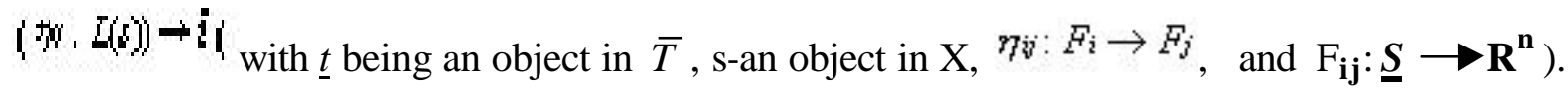
Thus, the time supercategory, $\bar{T}$, contains all intervals of time when transitions take place among distinct generating classes.

\section{Diagram 2:}

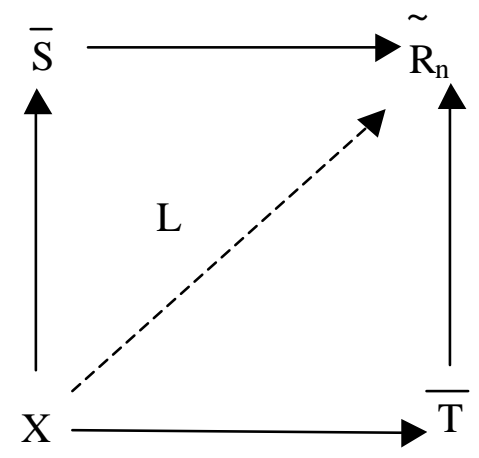

Qualitative Dynamics initiated by Poincaré (1881) is mainly concerned with problems of stability of dynamical systems. This theory introduces the notion of an attractor, $\mathrm{q}$ (stable equilibrium), which is a state, or a field of states such that the trajectory of any point near q goes to q, and no trajectory leaves q. An attractor is said to be structurally stable if any sufficiently small perturbation of the system leads to an attractor q' near the first one. The trajectories tending to attractors form the basins of the attractors. Basins of attractors may be intermingled, and in this case a conflict among attractors (Thom, 1969) arises. A biological example of a conflict among attractors follows. The phenomenon which is produced as a result of the penetration of many male pronuclei inside a single ovule is called polispermia. Each male nuclei would have a corresponding attractor in the state space of the whole system formed by the male pronuclei together with the ovule. The male pronucleus which penetrated first will have a corresponding dominating attractor because it begins first to orient trajectories towards it. The other attractors are then eliminated. Otherwise, the phenomenon of poliandria will be observed, that is, the fertilization of the female pronucleus by many male pronuclei. The presence of many conflicting 
attractors in the fertilized ovum leads to an unstable field which results in the abnormal growth and death of the organism developed from the ovum. However, a conflict among attractors may lead to metastable states as in the case of conflict between two inducing tissues of embryos. The regeneration of Planaria from "head," "body," or "tail" may also be explained in terms of attractors as follows.

Let us consider that there are three attractors corresponding respectively to "head," "tail," and "body." Any of the three attractors will regenerate the other two if they are connected as below,

\section{$\underline{\text { Diagram } 3}$}

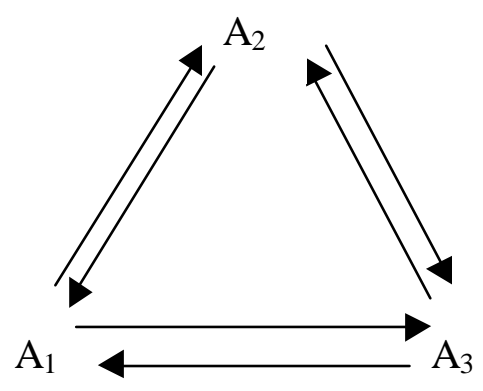

the arrows representing trajectories which go from the source attractor (say A1) and initiate the regeneration of the other attractor (say $\mathrm{A}_{2}$ or $\mathrm{A}_{3}$ ). This type of attractors will be called regenerating. The damage which induces regeneration will act as a perturbation on the unaffected attractor. It may be easily shown that the necessary and sufficient condition that a set of attractors would lead to stable fields of states is that they would form a pushout or a superpushout $[$ see (D6) of III].

If in our definition of a dynamical system we assign a set $\bar{I}$ of elements (called "inputs"), to each object of $\mathrm{X}$, and if we introduce an isomorphism FIX $\approx \mathrm{O}$, with $\mathrm{O}$ being a category of sets (the elements of which are called "outputs" of the system), then we obtain a correspondence among our definition and other definitions of a dynamical system as an input-output device. Let us 
denote the assignments defined above by $\mathrm{k}: \mathrm{ObX} \rightarrow \mathrm{I}$ and by $\mathrm{N}$ : FIX $\Rightarrow \mathrm{O}$. In a recent paper, Rosen suggested that the realization of a feedback would imply the decomposition of the state space of the system in two parts corresponding to a controller, and respectively to a controlled subsystem (Rosen, 1968b). The controller would be able to select its future inputs (coming from the controlled subsystem) by supplying appropriate outputs, that is, conforming to the data stored in its memory.

As an example consider that in the memory of the controller $\mathrm{K}$ there would exist a record of the fact that every time K sends monotonously decreasing outputs to the controlled subsystem, $\mathrm{K}$ receives a constant input. Then, in order to receive a constant input, $\mathrm{K}$ will produce monotonously decreasing outputs. If a constant input to $\mathrm{K}$ leads to transitions in a stable field of the state space of the whole system, $\mathrm{K}$ will easily succeed to ensure the stability of the system by supplying monotonously decreasing outputs to the controlled subsystem.

It was suggested by Rosen that in epigenetic regulatory mechanisms, positive feedbacks play a central role (Rosen, 1968b). In a positive feedback the controller acts in such a way as to receive continuously an enhanced response from the controlled subsystem. As a consequence, the activity of the system is sharply increased, and in technical systems it may lead to instabilities and to the destruction of the system. However, in the development of an organism from the ovum, some positive feedbacks have a converse effect. A stage of development of an organism cannot be considered as a state of the dynamical system, because the number of components of the system and its structure vary from one stage to the next. In our theory the change from a stage of development to another is represented as a change of the generating diagram at a moment $\bar{t}$ from $\bar{T}$. A positive feedback acting in the ovum leads to the continuous generation of new attractors, and correspondingly, to the formation of super-pushouts of attractors. As a result, the number of generating diagrams is continuously increased. One of the ways in which this is realized is the formation of new generating diagrams from the old, compatible generating diagrams. (The word "compatible" means here that the combinations obtained must not lead to the eventual death of the resulting organism.) This operation results in an increased number of relations. Thus, the presence of positive feedbacks in developmental processes seems to be implicitly contained in a principle which determines the course of 
development of an organismic set, and which was advanced by Rashevsky (1968b). However, there must exist a moment when the formation of new generating diagrams through the appearance of new cells and through differentiation processes cannot lead to a compatible combination.

In the course of development, as a result of the existence of many generating diagrams, many controllers are formed. Some of them begin to dominate the others, and the conflict among the corresponding attractors would lead to an inhibition of some dominated attractors. Otherwise, the compatibility condition will not be fulfilled and the organism will die. It may be that such incompatibility situations existed long ago in the formation of primary multicellular organisms. In normally developed organisms, the positive feedbacks are inhibited at the stage of maturation through a global negative feedback. However, the number of relations may continue to be increased through processes which take place inside the dominating controller. In the case of higher developed organisms, it is the brain that continues to increase the number of relations inside it.

\section{Relational Invariance, Analogy and Completion Laws.}

According to the principle of relational invariance (Rasheysky 1968a, c), there exists a mapping from the basic functional properties of higher developed organisms to the properties of an abstract "primordial" organism. This mapping is either an isomorphism or an epimorphism. The principle of relational invariance was also stated for regulatory mechanisms inside the same organism. In this form it states that there is a relational invariance of basic regulatory mechanisms of an organism. Thus, the mechanisms of control in the nervous system were shown to be isomorphic, or epimorphic, to the mechanisms of genetic control. An isomorphism between dynamical properties of two systems was previously called an analogy between the two systems (Rosen, 1968a).

However, there is no unique way to obtain knowledge on functional properties of more complicated organisms from the knowledge of functional properties of simpler organisms. Nevertheless, some procedures exist which can be applied in order to obtain the more 
complicated graph of properties of a higher organism from the more simple graph of a "primordial" one. Let us call these procedures 'completions'. In the theory of categories and functors, a completion of a category is the procedure through which one adds special objects, limits or colimits to a category. Then the properties of the new category are compared with those of the old category.

We suggest that there must exist completion laws - which are biologically significant, and which rule out improper completions (that is, completions which would lead to unreal organisms starting from a "primordial" organism).

Let us consider first the case of isomorphism between the sets of basic functional properties of two biological systems. The two systems will be called analogous. It may happen that the first biological system is characterized through a more reduced number of functional properties than the second. In this case, a monomorphism could be defined and this will be called a simple analogy of the two systems (see Rosen, 1968a).

If one can find a class of simple analogs of a given system which covers all properties of the system, then all dynamical properties of the given system may be defined in terms of the class of corresponding monomorphisms. Even more, if we consider instead of sets and monomorphisms, organismic supercategories and functors, then we can study dynamical properties of the more complicated organism by means of a study of the category of functors which define analogies.

According to the above introduced definitions, an organism will not be analogous with any of its stages of development but all its developmntal stages will be partially simply analogous to the mature stage. In order to make this idea more precise, a mathematical definition of analogy-which is formally different from that given by Rosen (1968a)-will be introduced here.

In our representation, two dynamical systems will be called analogous if there exists a leftadjoint functor $K: \bar{S} \rightarrow \bar{S}^{\prime}$, and an isomorphism $\bar{T} \rightarrow \bar{T}^{\prime}$. 

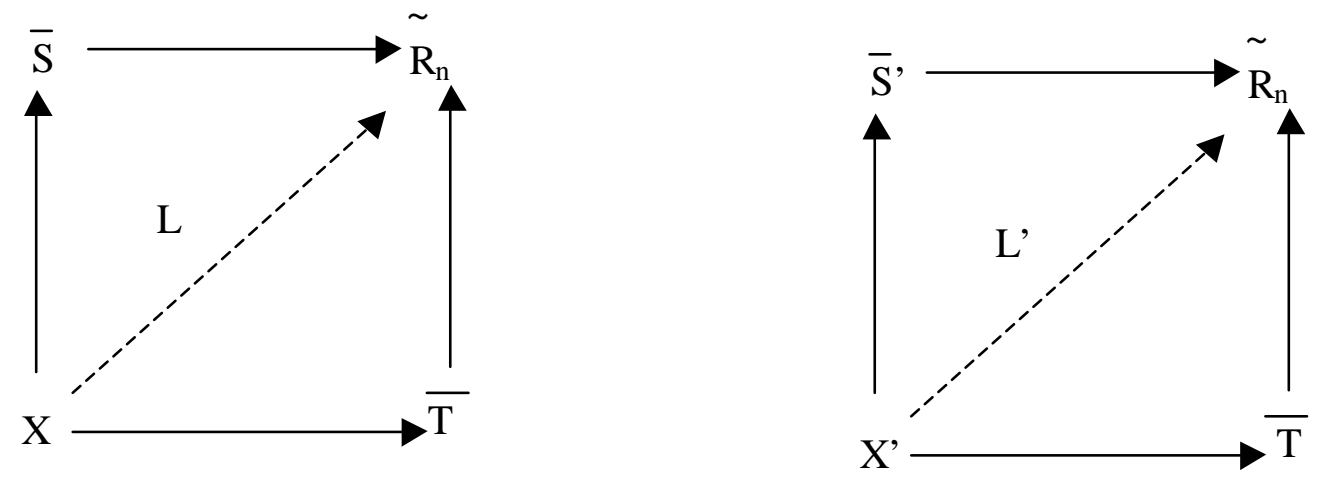

Diagram 4

The adjoint functor $K$, realizes a close comparison of the two systems as may be easily seen from its definition.

A covariant functor $F: C \rightarrow C^{\prime}$ is a left-adjoint of the functor $G: C^{\prime} \rightarrow C$, if for any couple $\left(\mathrm{X}, \mathrm{X}^{\prime}\right)$ of objects from a and $\mathrm{a}^{\prime}$, respectively, we are given a bijection $\mathbf{\phi}\left(\mathrm{X}, \mathrm{X}^{\prime}\right)$ :Homc $(\mathrm{X}$, $\left.\mathrm{G}\left(\mathrm{X}^{\prime}\right)\right) \rightarrow \operatorname{Hom}_{\mathrm{c}^{\prime}}\left(\mathrm{F}(\mathrm{X}), \mathrm{X}^{\prime}\right)$, such that for any morphism $\mathrm{f}: \mathrm{X} \rightarrow \mathrm{Y}$ of $\mathrm{C}$, and for any morphism $\mathrm{f}^{\prime}: \mathrm{X}^{\prime} \rightarrow \mathrm{Y}^{\prime}$ of $\mathrm{C}^{\prime}$, the following diagrams of sets and mappings are commutative:

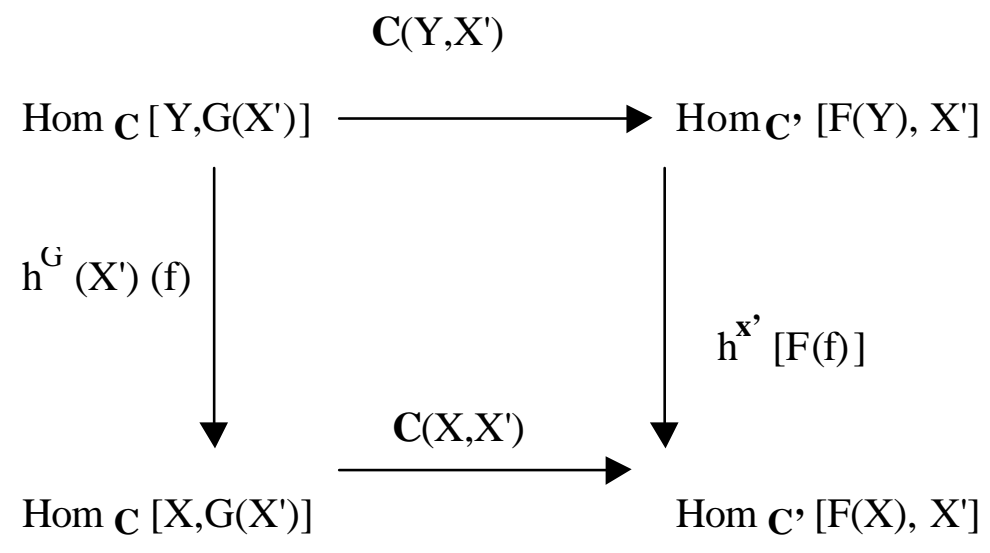

(5a) 


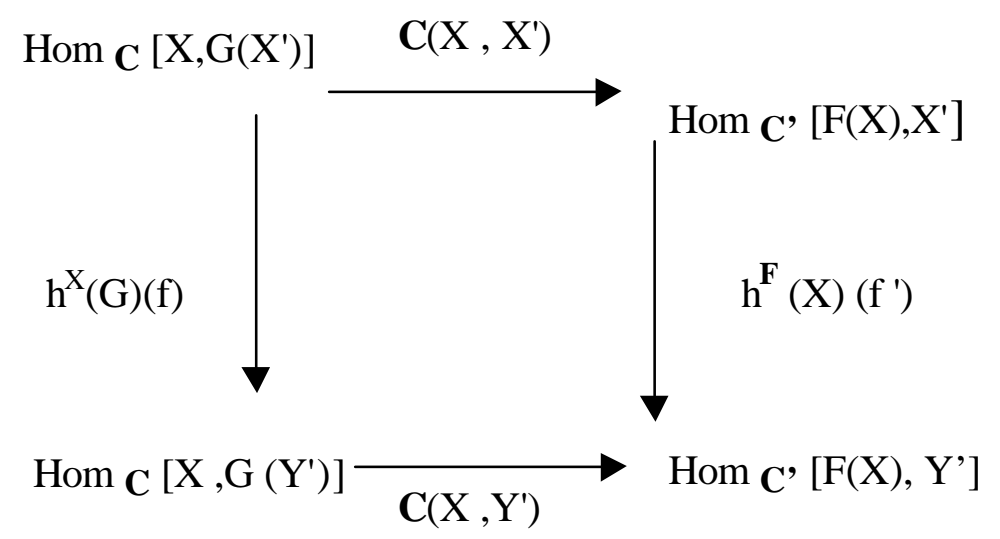

(5b)

Commutative

Diagrams 5a and 5b.

In the above diagrams we denoted by $\underline{\mathbf{h}}$ certain functors from an arbitrary category $\mathbf{C}$ to the category of sets, Ens. Their definitions are given below.

Let $\mathbf{C}$ be a category, and $\mathrm{X}$ an object in $\mathbf{C}$. The functor $\mathrm{h}^{\mathrm{X}}: \mathbf{C} \rightarrow$ Ens is defined by the following assignments $\mathrm{h}^{\mathrm{X}}(\mathrm{Y})=\operatorname{Hom} \mathbf{C}(\mathrm{X}, \mathrm{Y})$ for any $y \in O b C$ (with $\mathrm{X}$ a fixed object in $\mathrm{C}$ ) and $\mathrm{h}^{\mathrm{X}}(\mathrm{f}): \operatorname{Hom}_{\mathbf{C}} \rightarrow \operatorname{Hom}_{\mathbf{C}}(\mathrm{X}, \mathrm{Y}), \quad \operatorname{Hom}_{\mathbf{C}} \rightarrow \operatorname{Hom}_{\mathbf{C}}\left(\mathrm{X}, \mathrm{Y}^{\prime}\right)$, with $\mathrm{f:} \longrightarrow \mathrm{Y}^{\prime}$ being a morphism in $\mathbf{C}$. The last assignment is defined such as to have $\mathrm{h}^{\mathrm{X}}(\mathrm{f})(\mathrm{g})=\mathrm{f} \cdot \mathrm{g}$, with $\mathrm{g} \in \operatorname{Hom}_{\mathbf{C}}(\mathrm{X}, \mathrm{Y})$. The contravariant functor $\mathrm{h}$ is defined in a similar manner, such that $\mathrm{h}^{\mathrm{X}}(\mathrm{f})(\mathrm{g})=\mathrm{g}$ o f, for $\mathrm{g} \in \operatorname{Hom}_{\mathbf{C}}(\mathrm{X}, \mathrm{Y})$.

Two dynamical systems D and D' will be called simply analogous if there exists a leftadjoint functor M: $\bar{S} \rightarrow \bar{S}^{\circ}$ with $\bar{S}$ being the organismic supercategory in D and $\bar{S}^{\circ}$ being a

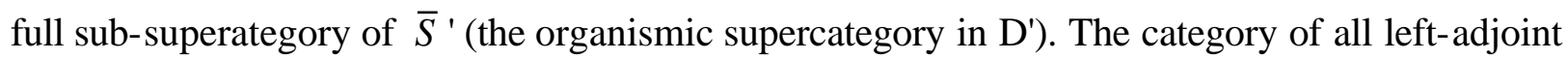
functors $\bar{S} \rightarrow \bar{S}^{\circ}$, with $\bar{S}^{\circ}$ fixed, and $\bar{S}$ varying in the class of analogs D sharing the dynamical properties of D' has a series of interesting features. In fact, these functors carry diagrams of linked observables of the first system into diagrams of linked observables of the second, precluding other possible assignments. Then, the organismic supercategory representing a 
"primordial" organism would play the role of an "initial object" in the metacategory of all organismic supercategories and left-adjoint functors. To complete this metacategory with a limit would be equivalent to supposing that there is an organism which has any other organism as a simple analog. This organism would be the highest that can possibly develop in a class of realizations of the corresponding abstract organismic supercategories.

The above mentioned completion determines the sense of biological evolution. Moreover, it results from this completion that the highest developed organism would be unique, up to an isomorphism. This isomorphism may stand for the basic functional properties of the most developed organism, and does not depend on particular realizations of the corresponding organismic supercategory (that is, the functionally isomorphic organisms may differ in their physicochemical structures).

\section{Quantum Automata and Relational Oscillations.}

Let us consider again the genetic system of a single cell. The genetic network of a cell was previously considered by Rashevsky (1967a) as an organismic set of order 0. As in an earlier paper, I shall represent in this section the genetic system as a quantum system, or as a quantum automaton. With the notations introduced in section $2^{\circ}$ a quantum automaton is defined as a particular kind of dynamical system Q whose states are all non-degenerate. If the morphism in Hom (obX $\mathrm{x} \bar{I}, \mathrm{obX})$ is represented by a quantum unitary operator $\mathbf{U}\left(\mathbf{t}, \mathbf{t}_{\mathbf{0}}\right)$, a transition in $\mathrm{X}$ corresponds to the following equation:

$$
\begin{aligned}
& \left|\psi_{t}\right\rangle=U\left(t, t_{0}\right)|\psi t 0\rangle \\
& \text {, with }|\psi t\rangle,|\psi t 0\rangle \text { states in } \mathrm{X} \text {. Now if } \\
& \mathrm{U}\left(\mathrm{t}, \mathrm{t}_{0}\right)=\exp -(\mathrm{i} / \hbar) \mathrm{H}\left(\mathrm{t}, \mathrm{t}_{0}\right)
\end{aligned}
$$

If $\mathrm{H}$ is the Hamiltonian operator of this system then one obtains the time dependent Schrödinger's equation of motion: 
$\partial|\psi\rangle=-(1 / \hbar) \mathrm{H}|\psi t\rangle$

Thus, one has the Schrödinger's representation in which states are functions of time whereas observables are independent of time (Sen, 1968). The operators $\mathbf{U}\left(\mathbf{t}, \mathbf{t}_{\mathbf{0}}\right)$ play the role of transition function from automata theory as far as they carry the couple (state, input) into the next state. If to each transition from $|\psi t 0\rangle_{\text {to a final state }}|\psi t\rangle_{\text {we }}$ assign a probability,

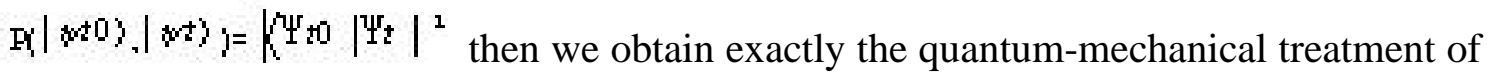
dynamical systems. Now, let us consider in this context the process of DNA duplication in a cell. Duplication may imply a number of repetitions of some basic quantum process in the course of sequential attachment of the new synthesized bases. The repeated process leads to the establishment of some relations among the components of the system, that is, among DNA, bases, and DNA-polymerase. These relations are also repeated in the course of duplication. If the repetition takes place at equal intervals of time we are to consider the whole system as $a$ relational oscillator, and the resulting process consists in relational oscillations. Similar situations may appear in the brain in the course of learning. Adaptive processes may be also considered as relational oscillations of a particular type. To conclude this section: relational oscillations in biological systems could be eventually mapped epimorphically onto relational oscillations of a quantum system. A particular case of the above-mentioned situation is the exact sequence of relational oscillators. If $\mathrm{A}, \mathrm{B}$, and $\mathrm{C}$ are three relational oscillators such that there exists a diagram in $\bar{C}$ which is the zero object - $\mathbf{0}$, of the generating supercategory $\bar{C}$, then an exact sequence is defined through the presence of the condition $\operatorname{Im} f=\operatorname{Ker} g$, with $f: A \rightarrow B$ and $g: B \rightarrow C$, such that $\operatorname{Ker} g=\{b \in a b \bar{B} \mid g(b)=0,0 \in O b \bar{C}\}, \operatorname{Im} f=f(A)$. The zero object of $\mathrm{G}$ is connected with all diagrams of observables in $\bar{C}$, and thus it may be an essential observable, or the diagram of essential observables in $\bar{C}$. Consequently, the dynamical properties of A are mapped epimorphically on some dynamical properties of B, and then on $\boldsymbol{a}$ single diagram of essential observables of $\bar{C}$. The three relational oscillators form a family of dynamically related systems, and will be called 'exactly homologous'. Having a knowledge of algebraic dynamical properties of a relational oscillator with a single essential observable one 
can derive algebraically some dynamical properties of exactly homologous relational oscillators with many essential observables. As an example, one may derive some properties of any group of operons from the properties of a single operon considered as a relational oscillator. Also, one could derive some properties of replicones, which initiate the duplication of DNA in cells of higher organisms, from the properties of a single replicone of bacteria. Nucleolar organizers of higher organisms would be related through an exact sequence with the nucleolar organizer of a unicellular organism, such as an Archea cell, or of an yeast cell.

\section{Oncogenesis, Dynamic Programming and Algebraic Geometry.}

In this section we shall discuss change s of normal controls in cells of an organism. On an experimental basis, we argued that some specific changes of cellular controls are produced in oncogenesis through an initial abnormal transfer of energy (Baianu, 1969a; Baianu and Marinescu, 1969b). Generally, the changes of controls in a cell may be produced through a strong localized perturbation of cellular activity (that is, through unusually strong forcing inputs), or through the prolonged action of unusual inputs. These changes become permanent if in one way or another, the activity of operons or replicons is impaired, that is, if a change of basic relational oscillators of the cell has taken place. In the current language of qualitative dynamics it may be translated as a change of dominating attractors, followed by the inhibition or destruction of the former dominating attractors. This kind of change is not necessarily a mutation, that is, the change may not produce the replacement of some essential observables in the genetic system. This may be the reason for which extensive research on cancer failed to discover so far a general, unique and specific alteration of the genetic system of cancer cells. The change of basic relational oscillators in the genetic system may have such consequences as, for example, abnormally large nucleoli. The reason may be that a change in the subspace of the controller produces the change of dynamic programming of the whole cell. Dynamic programming consists in the existence of distinguished states, or policies (Bellman, 1968) in the subspace corresponding to the controller, to which correspond specific changes of trajectories in the 
subspace of the controlled subsystem. The appropriate mathematical concept corresponding to such situations is found in Algebraic Geometry. The fact that some basic concepts of algebraic geometry are by now currently expressed in categorical terms, allows us to make use of the mathematical formalism of categories and functors. A projective space of $\underline{\mathbf{n}}$ dimensions will be assigned to the controlled subsystem, and a policy would be then represented by an allowable coordinate system in the projective space of the controlled subsystem. A projective space of $n$ dimensions is defined as a set of elements $S$ (called the points of the space) together with another set $\mathrm{Z}$ (the set of allowable coordinate systems in the space). Let $\left(a_{0}, \ldots, a_{n}\right)$ be an $n$-tuple of elements such that not all the elements $a_{0}, \ldots, a_{n}$ are zero. Two $n$-tuples $\left(a_{0}, \ldots, a_{n}\right),\left(b_{0}, \ldots, b_{n}\right)$ are said to be right-hand equivalent if there exists an element ? of a ground field such that ai $=$ bi?(i $=0, \ldots, n)$. A set of right-hand equivalent $(n+1)$-tuples is called a point of the right-hand projective number space of dimension $n$ over the ground field $\mathbf{K}$. The aggregate of such points is called a projective number space of dimension $\mathrm{n}$ over $\mathbf{K}$, and will be denoted by $\operatorname{PN}_{\mathrm{n}}(\mathbf{K})$. If $\mathrm{T}$ denotes a correspondence among the elements of a set $\mathrm{S}$ and the points of $\mathrm{PN}_{\mathrm{n}}(\mathrm{K})$, which is an isomorphism, then, to any element $\mathrm{A}$ of $\mathrm{S}$, there corresponds a set of equivalent $(n+1)$-tuples $\left(a_{0}, \ldots, a_{n}\right)$, where $T(A)$ is $\left(a_{0}, \ldots, a_{n}\right)$. Any $(n+1)$-tuple of this set is called a set of coordinates of A (Hodge and Pedoe, 1968). A set of equations written in matrix form as

$$
\mathbf{y}=\mathbf{A x}
$$

transforms $(\mathrm{n}+1)$-tuples $\left(\mathrm{x}_{0}, \ldots, \mathrm{x}_{\mathrm{n}}\right)$ into the set of equivalent $(\mathrm{n}+1)$-tuples $\left(y_{0} \lambda, \ldots, y_{\mathrm{n}} \lambda\right)$. That is, the equation (3) transforms a point of $\mathrm{PN}_{\mathrm{n}}(\mathrm{K})$ into a point of $\mathrm{PN}_{\mathrm{n}}(\mathrm{K})$. The set (3) of equations will be called a projective transformation of $\mathrm{PN}_{\mathrm{n}}(\mathrm{K})$ into itself. If $\mathrm{S}$ is the set from the definition of a projective space, then a projective transformation leads to a change of coordinate system in S. The different coordinate systems obtained through the application of different projective transformations are called allowable coordinate systems in S. Allowable coordinate systems in $\mathrm{S}$ define policies of the controller. In this case the set of all policies of a controller has the structure of a group as far as the projective transformations form a group. Now, if there is an extension $\mathrm{KO}$ of the ground field $\mathrm{K}$, and any $\mathrm{h}$ in $\mathrm{K}^{\mathrm{o}}$, $\mathrm{h}$ will be called algebraic if there exists a non zero polynomial $\mathrm{l}(\mathrm{x})$ in $\mathrm{K}[\mathrm{x}]$ such that $\mathrm{f}(\mathrm{h})=\mathrm{O}$. The aggregate of points defined by the set of equations 


$$
\mathbf{f}_{\mathbf{1}}\left(\mathbf{x}_{\mathbf{0}}, \ldots, \mathbf{x}_{\mathbf{n}}\right)=\mathbf{0},
$$

with $\mathrm{f}_{\mathrm{l}}\left(\mathrm{x}_{0}, \ldots, \mathrm{x}_{\mathrm{n}}\right)$ being a homogeneous polynomial over $\mathrm{K}$, is called an algebraic variety. Thus, one can define a dynamical program in terms of algebraic varieties of a projective space corresponding to the subspace of the controlled subsystem, and with allowable coordinate systems (projective transformations) corresponding to policies in the subspace of the controller. Analytical forms used in some economical problems are only examples of metric aspects of the qualitative theory of dynamical programming. This suggests that quantitative results concerning changes of controls in oncogenesis could be eventually obtained on the basis of algebraic computations by algebraic geometrical methods. The power of such computations and the elegance of the method is improved by means of the theory of categories and functors. A quantitative result which is directly suggested by this representation is the degree of synchrony in cultured cancer cells. However, this method of representation requires further investigation.

\section{Acknowledgements}

The author is indebted to Professor Nicholas Rashevsky for helpful suggestions. Also the author would like to thank Professor Robert Rosen for the sending of papers pertinent to this work. (updated by Ms. Hsiao Chen Lin, on May 20, 2004).

\section{REFERENCES}

1. Arbib, M. 1966. Categories of (M,R)-Systems. Bull. Math. Biophys., 28: 511-517

2. Ashby, W. R. 1960. Design for a brain, 2nd ed., New York: J. Wiley \& Sons, Inc.

3. Ashby, W. R 1956. .An Introduction to Oybernetics, New York: J. Wiley \& Sons, Inc.

4. Baianu, I.C. and Marinescu, M. 1968. Organismic Supercategories :I. Proposals for a General Unitary Theory of Systems. Bull. Math. Biophys., 30: 625-635.

5. Baianu, I. 1970. Organismic Supercategories: II. On Multistable Systems. Bull. Math. Biophys., 32: $539-561$.

6. Baianu, I. 1971. Organismic Supercategories and Qualitative Dynamics of Systems. Bull. Math. Biophys., 33: 339-354.

7. Baianu, I. 1971. Categories, Functors and Automata Theory., Proceed. 4th Intl. Congress LMPS, August-Sept. 1971.

8. Baianu, I. and Scripcariu, D. 1973. On Adjoint Dynamical Systems. Bull. Math. Biology., 35: 475-486. 
9. Baianu, I. 1973. Some Algebraic Properties of (M,R)-Systems in Categories. Bull. Math. Biophys, 35: $213-218$.

10. Baianu, I. and Marinescu, M. 1974. A Functorial Construction of (M,R)-Systems. Rev. Roum. Math. Pures et Appl ., 19: 389-392.

11. Baianu, I.C. 1977. A Logical Model of Genetic Activities in Lukasiewicz Algebras: The Non-Linear Theory., Bull. Math. Biology, 39:249-258.

12. Baianu, I.C. 1980. Natural Transformations of Organismic Structures. Bull. Math. Biology, 42: 431-446.

13. Baianu, I.C. 1980. Structural Order and Partial Disorder in Biological Systems. Bull. Math. Biology, 42: 464-468

14. Baianu, I.C.1983. Natural Transformations Models in Molecular Biology. SIAM Natl. Meeting, Denver, CO, USA.

15. Baianu, I.C. 1984. A Molecular-Set-Variable Model of Structural and Regulatory Activities in Metabolic and Genetic Systems ., Fed. Proc. Amer. Soc. Experim. Biol. 43: 917.

16. Baianu, I.C. 1987. Computer Models and Automata Theory in Biology and Medicine. In: "Mathematical Models in Medicine.",vol.7., M. Witten, Ed., Pergamon Press: New York, pp.1513-1577.

17. Bourbaki, N. 1958. Elements de Mathematique, Paris: Hermann \& Cle, Editeurs.

18. Carnap. R. 1938. "'The Logical Syntax of Language" New York: Harcourt, Brace and Co.

19. Cazanescu, D. 1967. On the Category of Abstract Sequential Machines. Ann. Univ. Buch., Maths \& Mech. series, $16(1): 31-37$.

20. Comorozan, S. and Baianu, I.C. 1969. Abstract Representations of Biological Systems in Supercategories.

Bull. Math. Biophys., 31: 59-71.

21. Ehresmann, Ch. 1966. "Trends Toward Unity in Mathematics." Cahiers de Topologie Et Geometrie Differentielle, 8,1-7.

22. Eilenberg, S. and S. MacLane. 1945. "General Theory of Natural Equivalences." Trans. .Am. Math. Soo., 58, $231-294$.

23. Eilenberg, S. and J. Wright. 1967. "Automata. in General Algebras." Seventy-Second Meeting American Math. Soc., 1-17.

24. Georgescu, G. and D. Popescu. 1968. "On Algebraic Categories." Rev. Roum. Math. Pures et Appl., 13, $337-342$.

25. Georgescu, G. and C. Vraciu 1970. "On the Characterization of Lukasiewicz Algebras." J Algebra, 16 (4), $486-495$.

26. Hilbert, D. and W. Ackerman. 1927. Grunduge.der Theoretischen Logik, Berlin: Springer.

27. Lawvere, F. W. 1963. "Functional Semantics of Algebraic Theories." Proc. Natl.Acad. Sci., 50, 869-872.

28. Lawvere, F. W. 1966. "The Category of Categories as a Foundation for Mathematics." In the Proc. Conf. Categorical .Algebra-La Jolla 1965, Eilenberg, S., et al. eds., Berlin, Heidelberg and New York: Springer-Verlag, pp. 1-20.

29. Lawvere, F. W. 1969. "Closed Cartesian Categories." (Lecture held as a guest of the Romanian Academy of 
Sciences).

30. Lofgren; L. 1968. "An Axiomatic Explanation of Complete Self-Reproduction." Bull.Math. Biophysics, 30, 317-348

31. Mitchell, B. 1965. The Theory of Categories. New York and London: Academic Press.

32. Rashevsky, N. 1954. "Topology and Life: In Search of General Mathematical Principles in Biology and Sociology." Bull. Math. Biophysics, 16, 317-348.

33. McCulloch, W and W. Pitts. 1943. "A logical Calculus of Ideas Immanent in Nervous Activity" Ibid., 5, 115133.

34. Pitts, W. 1943. "The Linear Theory of Neuron Networks” Bull. Math. Biophys., 5, 23-31.

35. Rosen, R.1958.a.”A relational Theory of Biological Systems” Bull. Math. Biophys., 20, 245-260.

36. Rosen, R. 1958b. "The Representation of Biological Systems from the Standpoint of the Theory of Categories" Bull. Math. Biophys., 20, 317-341.

37. Rosen, Robert. 1968. On Analogous Systems. Bull. Math. Biophys., 30: 481-492.

38..Rosen, Robert. 1973. On the Dynamical realization of (M,R)-Systems. Bull. Math. Biology., 35:1-10.

39. Russel, Bertrand and A.N. Whitehead, 1925. Principia Mathematica, Cambridge: Cambridge Univ. Press.

40. Warner, M. 1982. Representations of (M,R)-Systems by Categories of Automata., Bull. Math. Biol., 44:661-668. 